\title{
XXIX.
}

\section{Weiterer Beitrag zur Kenntnis der Stauungsblutungen nach Rumpfcompression.}

\author{
Von \\ Prof. Dr. Heinrich Braun in Göttingen.
}

(Mit Tafel III.)

\begin{abstract}
Seitdem ich in der medizinischen Gesellschaft in Göttingen am 6. Dezember 1900 eine dritte eigene Beobachtung von ausgedehnten Stauung:sblutungen nach Rumpfcompression mitgeteilt habe ${ }^{1}$ ), sind mir noch 2 weitere analoge Beobachtungen vorgekommen. Nur über den letzten der beiden Fälle möchte ich kurz berichten, hauptsächlich auch um ein Bild von dem Aussehen eines derartig Verletzten zu bringen.

Am 13. Mai 1904 wurde der 39 Jahre alte Landwirth Gustav H. ans Alrenborn, der in einer Kiesgrube in nach vorn übergebengter Stellung: stand, durch von hinten auf ihn einstürzende Erdmassen verschüttet, nach wenigen Minuten aber wieder ausgegraben. Nach dem Unfall war kein Elbrechen erfolgt and das Sensorium frei. Bei del einige Stunden nach der Verletzung erfolgten Aufnahme des Mannes in die chirurgische Klinik klagte derselbe über heftige Schmerzen in der Gegend der Schulterblätter und der Wirbelsäule, olne daß dort, oder an einer andern Körperstelle eine Verletzung gefunden werden konnte. Auffallend war jedoch die starke Anschwellung des Kopfes und die auf beiden Seiten, besonders aber rechts bestehende Protrusio bulbi. Die Lidspalten waren durch die stark angeschwollenen Augenlider fest geschlossen und nur gewaltsam zu öffnen, dabei zeigten sich die Conjunctivae durch Blutergüsse stark geschwellt, die Selerae mit Blut unterlaufen, das Sehvermögen normal, jedoch konnte zunächst eine ophthalmoskopische Untersuchung nicht vorgenommen werden. In dem blaurot aussehenden Gesicht fanden sich überall dicht zusammenstehende kleine und größere Blutungen, während zuf dem behaarten Kopfe dieselben nur in geringerer Zahl vorhanden waren. Ähnliche Ecchymosen zeigten sich auch in der Schleimhaut der Lippen, der Wangen und des weichen Gaumens. An dem Halse fanden sich bis auf einen etwa 21/2 cm breiten rings um den Hals herumlaufenden weißen Streifen - entsprechend
\end{abstract}

1) Ref. in Deutsche med. Wochenschr. 1901. Vereinsbeil. Nr. 2. S. 1 כ. 
dem Hemilenkragen - ebenfalls sehr zahlreiche Blutergiisse, die sich symmetrisch in dreieckiger Form auf die Brust erstreckten (rgl. Taf. III). Der Puls und die 'Temperatur waren normal, der Urin enthielt kein Eiweiß.

Der weitere Verlauf dieser Verletzungen bot keine Besonderheiten. Nach 3 Tagen war die Anschwellung der Augenlider bedeutend geringer geworden, so dab dieselben spontan wieder geöffuet werden konnten, der Exophthalmus fast vollkommen geschwunden und die Blutungen im Gesicht wesentlich abgeblaßt. Eine jetzt vorgenommene ophthalmoskopische Untersuchung zeigte keine Veränderungen im Auge, weder im Glaskörper, noch in der Netzhaut. Vom fünften Tage an verschwanden allmählich die Blıtmingen des Halses und der Brust; am achten Tage war die Hant des Gesichtes und des Halses wieder gleichmäßig, während die Conjunctiva bulbi et palpebrarum noch eine tiefrote, die Augenlider außen eine leicht bläuliche Verfärbung zeigten. $\Lambda \mathrm{m}$ 16. Mai wurde am rechten Bein eine isolierte Lähmung der Mm. peronaei festgestellt, zu der sich am folgenden Tage noch eine solche des M. tibialis anticus gesellte. Am 24. Juni konnte der Verletzte, der bis vor wenigen Tagen wegen einer doch möglichen Verletzung der Wirbelsäule im Bette gehalten war, aus der Klinik entlassen werden, die Blutungen im Gesicht und am Halse waren spurlos verschwunden, nur die Conjunctiva bulbi zeigte beiderseits, als letzten Rest der vorhanden gewesenen Blutung, noch eine leicht gelbliche Verfärbung. Die Lähmung des M. tibialis anticus am rechten Bein war vollkommen zurïckgegangen, während die Bewegungen der Mm. peronaei noch beträchtlich geschwächt erschienen.

Dieser Beobachtung möchte ich nur einige wenige Bemerkungen zur Ergänzung meiner früheren Mitteilungen über denselben Gegenstand hinzufügen.

Während Peirthes, der bekanntlich neuerdings zuerst die Aufmerksamkeit auf die uns hier interessierenden Erscheinungen am Kopfe, Halse, Rumpfe und an den oberen Extremitäten wieder gelenkt hat und sie als Folge eines starken Druckes auf den Thorax ansah, zeigte ich in meiner ersten Publikation 1) über diesen Gegenstand, daß dieselben ebenso auch durch Kompression des Unterleibes hervorgerufen werden können. Von früheren Beobachtungen waren mir damals nur diejenigen von Vogt und Hüter bekannt, die letzterer selbst kurz und Willers ausführlicher mitgeteilt hatte. Nach einer neueren Veröffentlichung von Pichler ${ }^{2}$ ) hat aber bereits schon Ollivier d'Angers 1837 diese multiplen Blutungen in der Gesichtshaut bei 23 Personen beobachtet, die bei einem Gedränge auf dem Marsfelde zu Paris um das Leben gekommen waren.

1) H. Braun, Über ausgedehnte Blutextravasate am Kopfe, Halse, Nacken und linken Arme infolge von Compression des Unterleibes. Deutsche Zeitschr. f. Chirurgie. 1899. 51. Bd. S. 599.

2) H.Pichler, Bemerkungen über "Staungsblutungen" insbesondere infolge eines epileptischen Anfalles. Zentraiblatt für innere Medizin. 1903. Nr. 4. 
Ferner hatte Tardieu 1) über analoge Blutungen verschiedenen Grades berichtet, die Hardy bei 8 Franen gefunden hatte, welche das Opfer eines blinden Feuerlärms geworden waren und Tardie ${ }^{2}$ ) selbst bei 8 Toten, die am Napoleonstage 1866 zu Paris an der Konkordiabrücke im Menschengedränge verunglückt waren.

In allen diesen Fällen hat wohl die durch äußere Gewalt hervorgerufene gleichzeitige Compression des Rumpfes diese Blutungen veranlaßt. Dali aber ohne eine solche, allein dureh starke Anspannung der Bauchmuskulatur ebenfalls die gleichen Blutungen hervorgerufen werden können, zeigen dieBeobachtungen von $\mathrm{W}$ ag en ma n ${ }^{3}{ }^{3}$, Pi chler (l. c.) und $\left.\mathrm{H} \circ \mathrm{p} \mathrm{p}^{\frac{1}{3}}\right)$. Ersterer hatte die Blutungen, die auffallend weit nach abwärts bis zum Nabel herabreichten, bei einem 19 Jahre alten Manne gesehen, der sich kräftig gegen einen auf seinen Rücken gefallenen Fahrstuhl gestemmt hatte. Da in diesem Falle der Mann bestimmt nicht durch diese Last gegen einen Gegenstand angedrückt wurde, so kann die Blutstauung nur durch eine ïbermäßige Anspannung der Bauchmuskulatur und durch Hinanfdrängen des Zwerehfells zu stande gekommen sein. Pichler hatte analoge Blutungen in der Gesichtshaut bei einem 25 jährigen Epileptiker beobachtet, bei dem ebenfalls nur die starke Anspannung der Muskulatur Schuld gewesen sein kann. Eine analoge Beobachtung hatte auch Tardieu gemacht. Hoppe sah die Blutungen bei einem Manne, der in vornübergebeugter Stellung heftig erbrochen hatte. Anzuschließen wären hier auch noch die aus gleicher Ursache zu stande kommenden Blutungen in der Bindebaut des Auges und der Hant des Gesichts, welche nach Tardieu bei Frauen bei langdauernden und schweren Entbindungen nicht selten sein sollen. Ob bei Keuchhusten außer den gewiß häufig vorkommenden Blutungen in die Conjunctiva und den später noch zu erwähnenden Blutungen in das Gehirn, manchmal auch solche in der Haut des Gesichtes und Halses vorkommen, ist mir nicht bekannt, aber wahrseheinlich.

Ohne auf alle einzelnen Erscheinungen, welche nach der Rumpfcompression bei dem zuletzt von mir beobachteten Kranken gefunden worden, einzugehen, möchte ich mir nur einige allgemeine Bemerkungen über die Blutungen in dem Auge und Gehirn und die Albuminurie erlauben. Auffallend stark war in diesem Falle die Protrusio bulbi, und

1) A. Tardiea, Mémoire sur la mort par suffocation Annales dhygiene publique et de méd. légale. 185 ว. 2. serie. T. IV. p. 371.

2) Derselbe, Ebenda. 1866. T. 26. p. 338.

3) Wagen mann, A., Multiple Blutungen der äußeren Haut und Bindehaut kombiniert mit eine: Netzhautblutung nach sehwerer Verletzung. Archiv der Ophth. von Gräfe. 1903. ว1.Bd. S. 550 .

4) Hoppe, Über multiple Gesichts- und Bindehautblutungen. Deutsche med. Wochenschr. 1901. Nr. 30. S. 505. 
sicherlich hat es sich dabei nicht um eine Täuschung gehandelt, die Pi chler für möglich hielt, der meinte, daß diese Erscheinnng zum Teil vorgetäuscht sein könnte, durch subkonjunktivale Blutergüsse, die Blutwülste bildeten. Wie ein früher von mir mitgeteilter Sektionsbefund beweist, kommen bei der Compression des Körpers Blutungen und Ödeme in das hinter dem Bulbus gelegene Fettgewebe vor, welche den Angapfel direkt nach vorn drängen. Mir scheint deshalb auch die Annahme von Hoppe nicht zutreffend, der den Exophthalmus als die Folge der übermäligen Dehnung der intraocularen Venennetze und der zahlreichen und erweiterten Anastomosen derselben mit den Gesichtsvenen ansieht. Blutungen im Innern des Auges wurden trotz genauster Untersuchung bei meinem letzten Kranken ebenso wie in den 3 früher von mir beobachteten Fällen nicht gefunden. Dieselben müssen überhaupt sehr selten sein und sind, soweit mir bekannt, bis jetzt nur von Wagenmann und $\mathrm{Neck}^{1}$ ) gefunden worden. Ersterer sah bei seinem Kranken eine Blutung in dem Hintergrund des rechten Auges, die so klein war, daß man anfangs über die Bedeutung des in der Netzhaut unterhalb der Pupille gefundenen kleinen senkrechten roten Streifens im Zweifel sein konnte, so daß erst am 2 Tage angegeben wird, daf es sich unzweifelhaft um eine kleine Blutung handelte, die in ihrer Ausdehnung unverändert geblieben und nur etwas dunkler geworden wäre. Bei dem von Neck beobachteten Kranken ergab die am 5. Tage nach der Verletzung vorgenommene ophthalmoskopische Untersuchung auf dem linken Auge kleine frische Blutungen in der Macula und im Glaskörper, deren Spuren noch nach 7 Monaten vorhanden waren.

Die öfters unmittellbar im Anschlub an die Verletzung festgestellten Sehstörungen werden demnach nicht durch materielle, sondern nur durch funktionelle Veränderungen der Netzhaut, welche die Folge der momentanen starken Blutstauung sein müssen, bedingt. Die Ursache für das Fehlen der Blutungen im Innern des Auges liegt gewiß in dem auf den kleinsten Gefäßen ruhenden intraocularen Druck. Hinzukommen soll nach den Auseinandersetzungen von Hoppe, der infolge der Rumpfompression nicht nur eine Steigerung. des venösen, sondern auch des arteriellen Blutdruckes annimmt, der hohe Widerstand, den die arterielle und venöse Blutwelle beim Eintritt in die Schädelkapsel finden, und durch den dieselben größtenteils rückläufig werden und nur mit sehr geringer Kraft in das Schädelinnere eintreten sollen.

In dem oben von mir mitgetheilten Falle fehlten ferner auch wieder

1) K. Neck, Über „Stauungsblutungen nach Rumpfcompression". Deutsche Zeitschr. f. Chirurgie. 1900. 57. Bd, S. 163. 
alle krankhaften Erscheinungen von seiten des Gehirns. Die Bewubtlosigkeit, welche bei manchen der Kranken erwähnt wird, ist gewil manchmal hervorgerufen durch die direkte Verletzung des Kopfes, die nicht ganz selten vorkommt, manchmal vielleicht auch durch den Schreck und den Shoc, welche die plötzliche schwere Verletzung hervorruft. Blutungen in das Gehirn scheinen nicht die Ursache für die Bewußtseinsstörungen zu sein, da sie sonst von längerer Dauer sein müßten. An und für sich wird man daraus, daß nach der Rumpfcompression keine Störungen der Gehirnfunktionen vorkommen, nicht schließen können, daß überhaupt keine Blutungen in die Gehirnsubstanz vorgekommen seien, da Blutungen in die Hirnhäute und in Gehirnteile, deren Verletzung keine Ausfallserscheinungen bedingen, ïberhaupt keine sichtbaren Erscheinungen hervorrufen werden. Die Möglichkeit des Vorkommens von Blutungen in das Gehirn durch starke Steigerung des Blutdruckes in der oberen Hohlvene scheint mir erwiesen durch die Beobachtungen, welche man bei Keuchhusten gemacht hat. Wiesinger ${ }^{1)}$ stellte in seiner Dissertation eine große Anzahl von Autopsien bei Personen, welche nach Keuchhusten gestorben waren, zusammen, in denen sowohl größere Blutergüsse, als auch zahlreiche capillare Blutungen in den Gehirnhäuten, in der Rinde und in der weiben Gehirnsubstanz gefunden wurden. Ein strikter Beweis dafür, daß bei der Compression des Rumpfes keine Gehirnblutungen vorkommen, kann nur durch Sektionsbefunde gegeben werden, und in dieser Hinsicht liegt die eine von mir früher gemachte Mitteilung vor, nach der trotz ausgedehnter Blutungen in die Haut des Gesichtes und Rumpfes, in die Schleimhaut der Mundhöhle, des Kehlkopfes, der Oberkiefer und Stirnhöhle bei der von Orth vorgenommenen genausten Sektion nicht die geringsten Blutungen in dem Gehirn oder in den Gehirnhäuten gefunden werden konnten. Auch Tardieu fand bei 2 Autopsien nicht die geringsten Blutungen im Gehirn, während Oll ivier d'Angers ${ }^{2}$ ) einmal blutige Flüssigkeit über einer Gehirnhemisphäre sah. Als Ursache für das Ausbleiben von Blutungen in die Schädelhı̈hle sah ich den intracraniellen Druck an. Neuerdings äußerte Orth ${ }^{3}$ ) gelegentlich einer in der medizinischen Gesellschaft in Berlin gemachten Vorstellung eines Falles von Staungsblutungen aus der v. Bergmann'schen Klinik durch Borchardt, daß ihn diese Annalıme nicht befriedige. Meiner Ansicht nach genügt die von mir gemachte Annahme, wenn wir sehen, daß sowohl der intraoculare Druck,

1) Wiesinger, Hirnblutungen bei Keuchhusten. Inaug.-Diss. Göttingen 1898.

21 Ollivier d'Angers, Annal. d'hygiène publ. et de méd. légale 1. serie. Т. XVIII, p. 485.

3) Berliner klin. Wochenschr. 1904. Nr. 25. S. 6\$3. Anmerkung. 
als awch der Gegendruck eines Hemdenkragens, oder eines anderen Kleidungsstückes, das in der Norm noch weit vom Halse absteht, genügt, um eine Blutung der Hautgefäßje an der Stelle, an welcher er anliegt, zu verhindern. Dahingestellt lasse ich es, ob nicht doch auch die Hirnsinus bei verschieden starkem Blutdruck eine verschiedene Menge Blutes aufzunehmen und dadurch auch zur Schwächung der Blutwelle beizutragen im stande sind. Nach der schon erwähnten Ansicht von Hoppe würde sich das Ausbleiben der Gehirnblutungen auch aus der geringen Kraft, mit welcher die venöse und arterielle Blutwelle in das Schädelinnere eindringen soll, erklären. Angenommen wird von Hoppe, wie dies früber auch schon von Willers geschehen war, då bei älteren Personen mit atheromatösen Gefäßen durch die infolge der Rumpfcompression eintretende Blutstauung eine Zerreißung der kleinen Gehirngefäße und der Tod eintreten könnte. Diese Annahme ist mir nicht wahrscheinlich, vor allem deshalb, weil bei dem fruher von mir mitgeteilten Sektionsbefunde eines 45 Jahre alten Mannes trotz der nachgewiesenen Verkalkung der Gehirngefäße doch keine Blutungen in dem Gehirn gefunden wurden. Jedenfalls sind bis jetzt keine Beobachtungen bekannt, aus denen die Richtigkeit dieser von Willers und Hoppe gemachten Annahme hervorgeht.

Noch besonders hervorheben möchte ich, dal auch bei dem zuletzt von mir beobachteten Verletzten trotz der sofort nach der Compression des Körpers vorgenommenen Untersuchung des Harns keine Albuminurie gefunden wurde. Dieselbe kam, soweit ich die Literatur kenne, überhaupt nur in meinem ersten Falle zur Beobachtung. Die Trübung. des Urins, welche Morian bei seinem Verletzten fand, kann nicht sicher als Folge der Gewalteinwirkung angesehen werden, da die Untersuchung nicht sofort nach der Verletzung, sondern erst später, nach mehrmaligem Katheterisieren des Kranken, vorgenommen wurde. Hoppe, der bei einer starken Kompression der Brust und des Bauches auch erhebliche Circulationsbeeinflussungen im Gebiete der unteren Hohlvene nicht für ausgeschlossen hält, meint, daß diese auch die Ursache für die vorübergehende Albuminurie ebenso wie für die von ihm bei seinem Kranken in der Nacht nach der Verletzung eingetretenen dünnen Stuhlentleerungen sein könnten.

Zuletzt nur noch ein Wort über die Bezeichnung der in Rede stehenden Verletzung. Perth es hatte die Bezeichnung: „Kopfblutungen nach Thoraxkompression" nicht für präzis genug gehalten, weil es sich auch um Blutergüsse in die Haut des Halses und der Brust handelte, und deshalb die Bezeichnung "Druckstauung“ kurzweg vorgeschlagen. 
Perthes selbst erachtete diese Bezeichnung aber nicht als ganz zutreffend, da durch dieselbe weniger die Blutstaung selbst, als der dadurch hervorgerufene Folgezustand bezeichnet würde. Zur Rechtfertigung der Einführung seines Vorschlags führt er die Gehirnerschütterung an, bei der es sich ebenso verhielte. Meiner Meinung nach soll man aber bei Einführung einer neuen Bezeichnung eine solche wählen, die möglichst prägnant ist, und es schien mir "Stauungs. blutungen nach Rumpfcompression" ") besser, weil darin zugleich die am meisten in die Augen fallende Erscheinung und auch die Ursache derselben enthalten ist; auch jetzt noch möchte ich deshalb diese Bezeichnung zur allgemeinen Annahme empfehlen. Gewiß wird auch jemand, der die nach einer Compression der Brust oder des Bauches entstehenden Symptome nicht kennt, sich eher bei der Bezeichnung Staungsisblutung eine Vorstellung von den zustande kommenden Erscheinungen machen können, als bei der Bezeichnung Druckstauung. In den späteren Publikationen wurde bald die eine, bald die andere Bezeichnung gewählt. Morian hält die Bezeichnung Stauungsblutungen nach Rumpfcompression für zu lang und $\mathrm{Hoppe}$ für nicht ganz zutreffend; letzterer meint, man würde besser von multiplen Hautblutungen durch plötzliche hohe Drucksteigerung in zusammenhängenden arteriellen und venösen Bahnen sprechen. Dem gegenüber ist hervorzuheben, daf man bei der Annahme des Wortes "Druckstauung" doch auch hinzufügen mübte, durch was dieselbe verursacht wurde. Einseitig wäre es, diese Bezeichnung nur für die durch Compression des Rumpfes zustande kommenden Blutungen anzuwenden, nicht aber auch für solche, die nach starker Anspannung der Bauchmuskulatur, bei Epilepsie, bei Keuchhusten usw. eintreten, die ihrem Wesen nach doch alle als gleichwertig angesehen werden müssen. Gegenüber $\mathrm{H}$ oppe möchte ich nur hervorheben, dah seine Bezeichnung, abgesehen von der Länge, auch insofern ungenau ist, als man gelegentlich, wenn auch gewiß selten, die gleichen Blutungen in Fällen findet, in denen es sich bestimmt nur um Staung des Blutes in den Venen handelt, z. B. nach Anlegung einer elastischen Binde zur Herbeiführung der Blutstaung nach Bier. Vor etwa $1 / 2$ Jahre sah ich bei einem 37 jährigen Arbeiter, dem wegen verzögerter Callusbildung ein gefensterter Gipsverband gemacht war, daß nach $\Lambda$ nlegung einer Gummibinde zwischen derselben und dem Anfange des Gipsverbandes zikulär um das Bein unzählige, dicht gedrängt stehende kleinste Blutergüsse in die blaurot verfärbt aussehende Haut hinein zustande gekommen waren

1) H. Braun, Staungsblutungen nach Rumpfcompression. Deutsche Zeitschr. f. Chirurgie. 1900. 56. Bd. S. 184. 


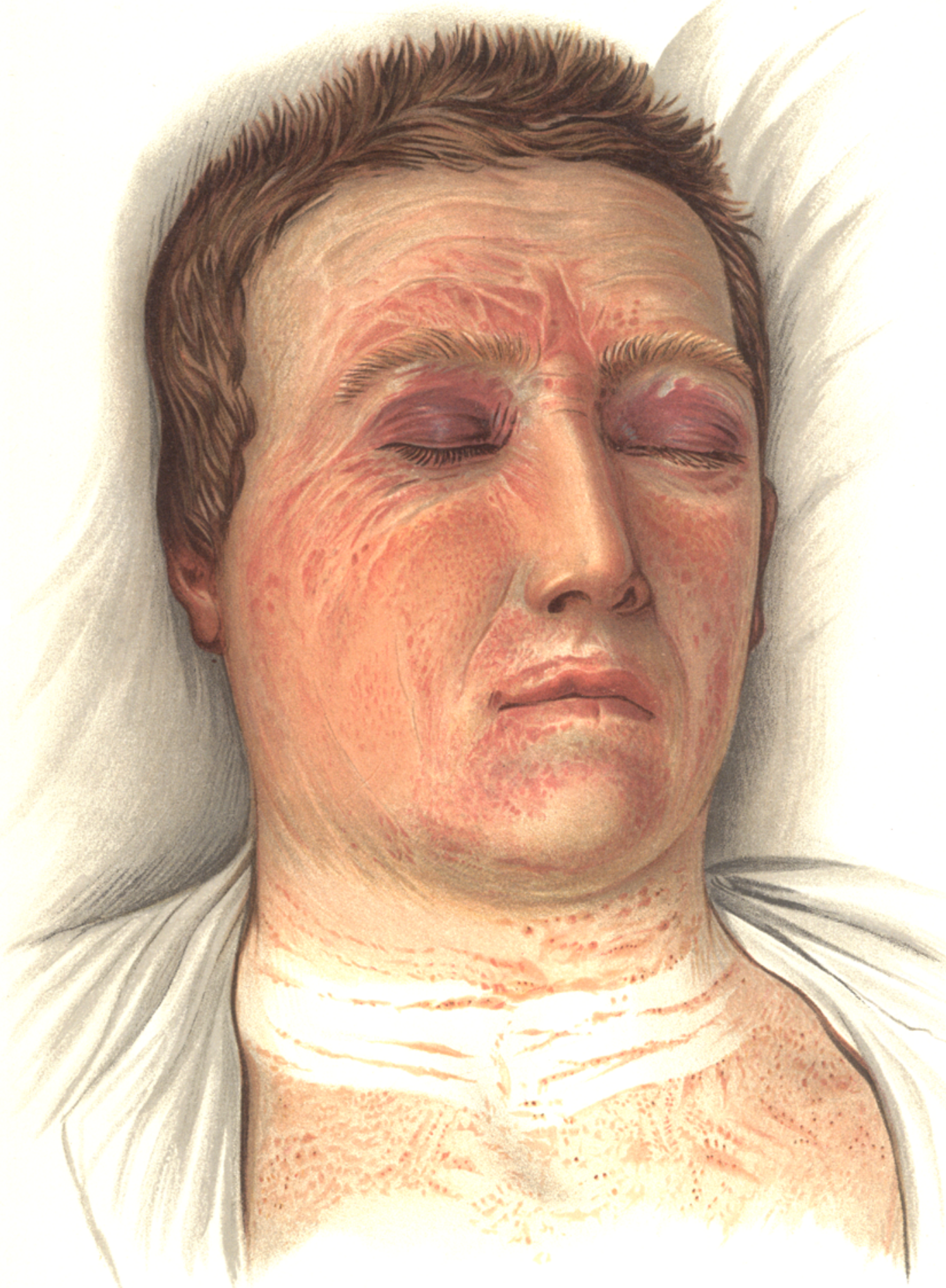

